\title{
Prognostic Significance of The Neutrophil-To- Lymphocyte Ratio in Patients With Non-Muscle Invasive Bladder Cancer Treated With Intravesical Bacillus Calmette-Guérin and The Relationship With The CUETO Scoring Model
}

Jin Woo Kim

Kyungpook National University

Jae-Wook Chung

Kyungpook National University

Eun Hye Lee

Kyungpook National University Hospital

So Young Chun

Kyungpook National University Hospital

Dong Jin Park

Dongguk University School of Medicine

Kyeong Hyeon Byeon

Kyungpook National University

Seock Hwan Choi

Kyungpook National University

Jun Nyung Lee

Kyungpook National University Hospital

Bum Soo Kim

Kyungpook National University Hospital

Hyun Tae Kim

Kyungpook National University

\section{Eun Sang Yoo}

Kyungpook National University

Tae Gyun Kwon

Kyungpook National University

Yun-Sok Ha ( $\nabla$ yunsokha@gmail.com )

Kyungpook National University

Tae-Hwan Kim

Kyungpook National University 


\section{Research Article}

Keywords: Neutrophil-to-lymphocyte ratio, Bacillus Calmette-Guérin, Club Urológico Español de Tratamiento Oncológico risk model, Survival

Posted Date: December 8th, 2020

DOl: https://doi.org/10.21203/rs.3.rs-116331/v1

License: (c) (i) This work is licensed under a Creative Commons Attribution 4.0 International License.

Read Full License 


\section{Abstract}

Background: The neutrophil-to-lymphocyte ratio (NLR) is a marker of the systemic inflammatory response, which is associated with tumor recurrence and progression. In this study, we evaluated the predictability of a modified Club Urológico Español de Tratamiento Oncológico (CUETO) scoring model and preoperative NLR in patients with non-muscle invasive bladder cancer (NMIBC).

Methods: From August 2005 to May 2016, a total of 281 patients received intravesical bacillus CalmetteGuérin therapy after transurethral resection of a bladder tumor. The pathologic stage of all patients was Ta or T1. Multivariate Cox regression analysis and Kaplan-Meier curves were used to evaluate the prognosis predictability of the CUETO risk model and NLR. Of 281 patients, 84 (29.9\%) experienced recurrence and 14 (5.0\%) developed progression. The mean follow-up period was 46 months. The cut-off value for NLRs was 2.29 .

Results: Of the study patients, 108 (38.4\%) displayed a high NLR (> 2.29). In Kaplan-Meier curve analysis, a high NLR was associated with lower recurrence-free survival (RFS) $(P<0.001)$ and progression-free survival (PFS) $(P=0.002)$. CUETO scores were associated with RFS $(P<0.001)$, but not with PFS $(P=0.423)$. A combination of NLRs and the CUETO risk model correlated with RFS $(P<0.001)$ and PFS $(P=0.002)$. In multivariate analysis, female gender, concomitant carcinoma in situ (CIS), tumor number $>3$, recurrent tumors, and a high NLR were independent factors predicting recurrence (all $\mathrm{P}<$ 0.05). Concomitant CIS, recurrent tumors, and a high NLR were independent factors for predicting progression (all $\mathrm{P}<0.05$ ).

Conclusion: In patients with NMIBC, an NLR >2.29 was identified as a significant factor for predicting tumor recurrence and progression. Inclusion of preoperative NLR enhanced the accuracy of the CUETO model to predict disease progression. We therefore recommend that patients with a high NLR receive more aggressive management.

\section{Background}

The most common malignant tumor of the urinary tract is bladder cancer, and the fourth-most common cancer among males in developed countries [1]. Three-quarters of bladder cancer patients are diagnosed with non-muscle invasive bladder cancer (NMIBC), which includes Tis, Ta and T1 pathologic stages [2]. Transurethral resection of bladder tumor (TUBRT) is a primary surgical treatment used to treat patients with NMIBC [3]. After initial TURBT, immunotherapy with intravesical instillation of bacillus CalmetteGuérin (BCG) is the most effective adjuvant therapy for intermediate- and high-risk NMIBC [4]. Despite the effectiveness and safety of BCG, recurrence rates is $32.6-42.1 \%$ and progression rates is $9.5-13.4 \%$ [5].

The major treatment challenge with NMIBC is preventing progression to muscle invasive bladder cancer (MIBC), which rapidly worsens prognoses [6]. Thus, it is significant to predict risk factors for disease recurrence and progression in NMIBC patients according to individual characteristics, including pathology and choose optimal treatment modalities to enhance oncologic outcomes. 
To predict recurrence of NMIBC and progression to MIBC, numerous clinical and pathological factors are commonly used to assign patients to different risk groups. Of these risk models, a scoring model developed by the Club Urológico Español de Tratamiento Oncológico (CUETO, or Spanish Urological Club for Oncological Treatment) is considered the most reliable. The CUETO model was developed as a riskscoring tool that predicts the probability of disease recurrence and progression in BCG-treated patients at 1,2 , and 5 years [7]. It is now recognized that increased systemic inflammatory responses induced by tumor microenvironments trigger alteration of acute-phase reactive substance and hematologic ingredient [8]. Among these serum markers are neutrophil and lymphocyte counts, which can indicate relative neutrophilia and lymphocytopenia. In various tumor patients, a higher percentage of neutrophils than lymphocyte is associated with reduced cancer-free and overall survival [9-11].

We evaluated the efficiency of a modified CUETO scoring model combined with preoperative NLRs to predict recurrence and progression of disease in NMIBC patients.

\section{Methods}

\section{Ethics statement}

This study was approved by the institutional review board of Kyungpook National University, Hospital, Daegu, Republic of Korea (IRB Number KNUH 2020-03-042). The study was carried out in agreement with the applicable laws and regulations, good clinical practices, and ethical principles as described in the Declaration of Helsinki. The institutional review board of Kyungpook National University Chilgok Hospital waived because of the retrospective nature of the study.

\section{Study design}

The study was a single institution, retrospective observational trial. From August 2005 to May 2016, a total of 281 patients who underwent BCG induction therapy after complete TURBT were included in this study. They had not previously received intravesical BCG and showed no side effects of BCG or signs of recurrence during BCG therapy. All patients were diagnosed as histologically Ta or T1. Patients with only carcinoma in situ (CIS) were excluded, as were those found to have advanced bladder or ureteral tumors or non-urothelial carcinoma at the first TUR. Patients with hematologic malignance and acute or chronic infection were also excluded.

Preoperative NLR was calculated by a complete blood count with differential. The cut-off NLR value was set at 2.29 , according to a receiver operating characteristic curve (sensitivity: $59.5 \%$, specificity: $69.5 \%$ ) (Fig. 1).

The follow-up period of patients was calculated from the first TURBT to the last cystoscopy examination. We performed urine cytology, cystoscopy, chest X-ray, and computed tomography (CT) scans for followup study. During the first year after TURBT, follow-up study was conducted at 3,6 , and 12 months. Cystoscopy and urine cytology were performed every 6 months until 2 years after TURBT, and yearly 
thereafter. Imaging analyses, including chest X-ray and CT scans were examined every 6 months from 1 to 5 years, and annually thereafter.

Recurrence of disease was defined as a newly pathological confirmed bladder cancer regardless of stage after completion of BCG induction therapy. Progression of disease was defined as from Ta or T1 to stage T2 or higher disease (MIBC).

\section{Intravesical bacillus Calmette-Guérin instillation}

In all patients, BCG tice strain $12.5 \mathrm{mg}$ (Oncotice) was used. A BCG suspension with $50 \mathrm{~mL}$ of $0.9 \%$ normal saline was instilled to the bladder via a $10 \mathrm{Fr}$ urethral catheter. Patients were advised not to urination for two hours. Induction BCG therapy was initiated 2 weeks after TUR and repeated once a week for 6 weeks. We did not perform BCG maintenance therapy.

\section{Club Urológico Español de Tratamiento Oncológico scoring model}

Scoring tumor recurrence and progression were calculated according to the CUETO scoring model, which includes age, gender, previous recurrence status, tumor stage (2002 TNM classification) and grade, multiplicity, and concomitant CIS. In accordance with the 2004 World Health Organization (WHO) grading system, we classified tumor grades as low or high [12].

\section{Statistical analysis}

Non-continuous variables of patient characteristics, including gender, T stage, tumor grade, concomitant CIS, size, number, recurrence status and CUETO risk model were analyzed using the chi-square test.

Student's t-test was used to analyze continuous variables such as age, body mass index (BMI) and follow up periods. In addition, a multivariable Cox regression model was used for analysis of tumor recurrence and progression, and Kaplan-Meier curves via a log-rank test were used for analysis of recurrence-free survival (RFS) and progression-free survival (PFS). Statistical analysis was performed using SPSS 16.0 for Windows (SPSS Inc., Chicago, IL, USA), and a P value $<0.05$ was considered statistically significant.

\section{Results}

Table 1 lists the characteristics of patients with NMIBC. A total of 173 patients had an NRL $\leq 2.29$ $(61.6 \%)$ and 108 (38.4\%) had an NLR > 2.29. Eighty-four patients (29.9\%) experienced recurrence and 14 (5.0\%) showed progression after TUR followed by BCG therapy. Female patients accounted for $12.8 \%$ $(36 / 281)$ of the study groups. The mean age was $67.38 \pm 10.58$ years. Mean BMI was $23.88 \pm$ $3.37 \mathrm{~kg} / \mathrm{m}^{2}$. The mean follow-up period was $46.65 \pm 20.80$ months. No significant differences were evident in gender, age, BMI, and follow-up periods between the two groups. T stage analysis after TUR found 105 patients (37.4\%) were in Ta category patients 176 patients $(62.6 \%)$ were T1. High-grade tumors were found in 251 patients (89.3\%). Concomitant CIS was shown in 24 patients (8.5\%). Tumor greater than $3 \mathrm{~cm}$ in size were found in 86 patients (30.6\%). A tumor number greater than 3 was shown in 
66 patients (23.5\%). Recurrent tumors accounted for $14.6 \%(41 / 281)$ of the patients. There were no significant differences in tumor stage, grade, concomitant CIS, size, number, and prior recurrence status between the two groups. The mean CUETO score according to recurrence and progression was $5.58 \pm$ 2.13 and $8.27 \pm 2.26$, respectively. No significant differences were found in categorical or non-categorical CUETO scores between the two groups. 
Table 1

Characteristics of patients with NMIBC

\begin{tabular}{|c|c|c|c|c|}
\hline & $\begin{array}{l}\text { Total } \\
\mathrm{n}=\mathbf{2 8 1}\end{array}$ & $\begin{array}{l}\text { NRL } \leq \\
2.29 \\
n=173\end{array}$ & $\begin{array}{l}\text { NLR }>2.29 \\
n=108\end{array}$ & $\begin{array}{l}P \text { - } \\
\text { value }\end{array}$ \\
\hline Recurrence & $84(29.9 \%)$ & $35(20.2 \%)$ & $49(45.4)$ & $\begin{array}{l}< \\
0.001\end{array}$ \\
\hline Progression & $14(5.0 \%)$ & $3(1.7 \%)$ & $11(10.2 \%)$ & 0.002 \\
\hline Gender & & & & 0.952 \\
\hline Male & $\begin{array}{l}245 \\
(87.2 \%)\end{array}$ & $\begin{array}{l}151 \\
(87.3 \%)\end{array}$ & $94(87.0 \%)$ & \\
\hline Female & $36(12.8 \%)$ & $22(12.7 \%)$ & $14(13.0 \%)$ & \\
\hline Age, years & $\begin{array}{l}67.38 \pm \\
10.58\end{array}$ & $\begin{array}{l}68.00 \pm \\
9.87\end{array}$ & $\begin{array}{l}66.40 \pm \\
11.60\end{array}$ & 0.218 \\
\hline Body mass index, $\mathrm{kg} / \mathrm{m}^{2}$ & $\begin{array}{l}23.88 \pm \\
3.37\end{array}$ & $\begin{array}{l}23.94 \pm \\
3.07\end{array}$ & $\begin{array}{l}23.79 \pm \\
3.81\end{array}$ & 0.727 \\
\hline Follow-up periods, months & $\begin{array}{l}46.65 \pm \\
20.80\end{array}$ & $\begin{array}{l}45.25 \pm \\
19.14\end{array}$ & $\begin{array}{l}48.89 \pm \\
23.13\end{array}$ & 0.173 \\
\hline T stage & & & & 0.239 \\
\hline $\mathrm{Ta}$ & $\begin{array}{l}105 \\
(37.4 \%)\end{array}$ & $60(34.7 \%)$ & $45(41.7 \%)$ & \\
\hline T1 & $\begin{array}{l}176 \\
(62.6 \%)\end{array}$ & $\begin{array}{l}113 \\
(65.3 \%)\end{array}$ & $63(58.3 \%)$ & \\
\hline Tumor grade & & & & 0.327 \\
\hline Low & $30(10.7 \%)$ & $16(9.2 \%)$ & $14(13.0 \%)$ & \\
\hline High & $\begin{array}{l}251 \\
(89.3 \%)\end{array}$ & $\begin{array}{l}157 \\
(90.8 \%)\end{array}$ & $94(87.0 \%)$ & \\
\hline $\begin{array}{l}\text { Concomitant } \\
\text { carcinoma in situ }\end{array}$ & & & & 0.098 \\
\hline No & $\begin{array}{l}257 \\
(91.5 \%)\end{array}$ & $\begin{array}{l}162 \\
(93.6 \%)\end{array}$ & $95(88.0 \%)$ & \\
\hline Yes & $24(8.5 \%)$ & $11(6.4 \%)$ & $13(12.0 \%)$ & \\
\hline Tumor size & & & & 0.779 \\
\hline$\leqq 3 \mathrm{~cm}$ & $\begin{array}{l}195 \\
(69.4 \%)\end{array}$ & $\begin{array}{l}119 \\
(68.8 \%)\end{array}$ & $76(70.4 \%)$ & \\
\hline
\end{tabular}




\begin{tabular}{|c|c|c|c|c|}
\hline & $\begin{array}{l}\text { Total } \\
\mathrm{n}=\mathbf{2 8 1}\end{array}$ & $\begin{array}{l}\mathrm{NRL} \leq \\
2.29 \\
\mathrm{n}=173\end{array}$ & $\begin{array}{l}\text { NLR }>2.29 \\
n=108\end{array}$ & $\begin{array}{l}P \\
\text { value }\end{array}$ \\
\hline$>3 \mathrm{~cm}$ & $86(30.6 \%)$ & $54(31.2 \%)$ & 32 (29.6\%) & \\
\hline Tumor number & & & & 0.293 \\
\hline$\leqq 3$ & $\begin{array}{l}215 \\
(76.5 \%)\end{array}$ & $\begin{array}{l}136 \\
(78.6 \%)\end{array}$ & 79 (73.1\%) & \\
\hline$>3$ & $66(23.5 \%)$ & $37(21.4 \%)$ & 29 (26.9\%) & \\
\hline Recurrent tumor & & & & 0.792 \\
\hline No & $\begin{array}{l}240 \\
(85.4 \%)\end{array}$ & $\begin{array}{l}147 \\
(85.0 \%)\end{array}$ & 93 (86.1\%) & \\
\hline yes & $41(14.6 \%)$ & $26(15.0 \%)$ & 15 (13.9\%) & \\
\hline $\begin{array}{l}\text { CUETO score according to recurrence (non- } \\
\text { categorical) }\end{array}$ & $5.58 \pm 2.13$ & $5.57 \pm 2.08$ & $5.59 \pm 2.20$ & 0.938 \\
\hline $\begin{array}{l}\text { CUETO score according to recurrence } \\
\text { (categorical) }\end{array}$ & & & & 0.826 \\
\hline $1 \sim 4$ & 92 (32.7\%) & $55(31.8 \%)$ & 37 (34.3\%) & \\
\hline $5 \sim 6$ & $\begin{array}{l}106 \\
(37.7 \%)\end{array}$ & $67(38.7 \%)$ & 39 (36.1\%) & \\
\hline $7 \sim 9$ & $66(23.5 \%)$ & $42(24.3 \%)$ & $24(22.2 \%)$ & \\
\hline $10 \sim 16$ & $17(6.1 \%)$ & $9(5.2 \%)$ & $8(7.4 \%)$ & \\
\hline $\begin{array}{l}\text { CUETO score according to progression (non- } \\
\text { categorical) }\end{array}$ & $8.27 \pm 2.26$ & $8.35 \pm 2.20$ & $8.15 \pm 2.37$ & 0.462 \\
\hline $\begin{array}{l}\text { CUETO score according to progression } \\
\text { (categorical) }\end{array}$ & & & & 0.453 \\
\hline $1 \sim 4$ & $22(7.8 \%)$ & $12(6.9 \%)$ & $10(9.3 \%)$ & \\
\hline $5 \sim 6$ & $42(15.0 \%)$ & $22(12.7 \%)$ & 20 (18.5\%) & \\
\hline $7 \sim 9$ & $\begin{array}{l}120 \\
(42.7 \%)\end{array}$ & $78(45.1 \%)$ & 42 (38.9\%) & \\
\hline $10 \sim 14$ & 97 (34.5\%) & $61(35.3 \%)$ & $36(33.3 \%)$ & \\
\hline
\end{tabular}

Table 2 shows a multivariate Cox proportional hazards regression model for predicting recurrence. Female gender, concomitant CIS, multiplicity ( $>3)$, prior recurrence status and an NLR $>2.29$ were independent prognostic factors for tumor recurrence (hazard ratio [HR], 2.565; 95\% confidence internal [Cl], 1.485-4.431; P = $0.001 / \mathrm{HR}, 2.007 ; 95 \% \mathrm{Cl}, 1.059-3.802 ; \mathrm{P}=0.033 / \mathrm{HR}, 1.874 ; 95 \% \mathrm{Cl}, 1.165-3.012$; 
$P=0.010 / \mathrm{HR}, 2.320 ; 95 \% \mathrm{Cl}, 1.280-4.207 ; \mathrm{P}=0.006 / \mathrm{HR}, 2.451 ; 95 \% \mathrm{Cl}, 1.567-3.834 ; \mathrm{P}<0.001$, respectively).

Table 2

Multivariate Cox proportional hazards regression model for predicting recurrence

\begin{tabular}{|c|c|c|c|c|}
\hline & \multirow[t]{2}{*}{ HR } & \multicolumn{2}{|l|}{$95 \% \mathrm{Cl}$} & \multirow[t]{2}{*}{$P$-value } \\
\hline & & Lower & Upper & \\
\hline Age & 1.022 & 0.999 & 1.047 & 0.065 \\
\hline Gender (male vs. female) & 2.565 & 1.485 & 4.431 & 0.001 \\
\hline T stage (Ta vs T1) & 1.576 & 0.911 & 2.725 & 0.104 \\
\hline Tumor grade (low vs high) & 1.226 & 0.541 & 2.780 & 0.625 \\
\hline Presence of carcinoma in situ (no vs. yes) & 2.007 & 1.059 & 3.802 & 0.033 \\
\hline Multiplicity ( $\leqq 3$ vs $>3$ ) & 1.874 & 1.165 & 3.012 & 0.010 \\
\hline Tumor size ( $\leqq 3$ vs $>3$ ) & 1.267 & 0.793 & 2.023 & 0.322 \\
\hline Prior recurrence status (no vs. yes) & 2.320 & 1.280 & 4.207 & 0.006 \\
\hline $\operatorname{NLR}(\leqq 2.29$ vs. $>2.29)$ & 2.451 & 1.567 & 3.834 & $<0.001$ \\
\hline
\end{tabular}

Table 3 shows a multivariate Cox proportional hazards regression model for predicting progression. Concomitant CIS, prior recurrence status and an NLR $>2.29$ were independent prognostic factors for progression (HR, 4.903; 95\% Cl, 1.523-15.782; $\mathrm{P}=0.008 / \mathrm{HR}, 3.997 ; 95 \% \mathrm{Cl}, 1.175-13.602 ; \mathrm{P}=0.027 /$ $\mathrm{HR}, 5.911 ; 95 \% \mathrm{Cl}, 1.579-22.126 ; \mathrm{P}=0.008$, respectively). 
Table 3

Multivariate Cox proportional hazards regression model for predicting progression.

\begin{tabular}{|c|c|c|c|c|}
\hline & \multirow[t]{2}{*}{ HR } & \multicolumn{2}{|l|}{$95 \% \mathrm{Cl}$} & \multirow[t]{2}{*}{ P-value } \\
\hline & & Lower & Upper & \\
\hline Age & 1.041 & 0.973 & 1.114 & 0.246 \\
\hline Gender (male vs female) & NA & NA & NA & 0.981 \\
\hline T stage (Ta vs $\mathrm{T} 1)$ & 1.184 & 0.314 & 4.441 & 0.802 \\
\hline Tumor grade (low vs high) & 3.071 & 0.320 & 29.513 & 0.331 \\
\hline Presence of carcinoma in situ (no vs. yes) & 4.903 & 1.523 & 15.782 & 0.008 \\
\hline Multiplicity ( $\leqq 3$ vs $>3$ ) & 1.762 & 0.518 & 5.987 & 0.364 \\
\hline Tumor size ( $\leqq 3$ vs > 3 ) & 2.077 & 0.645 & 6.692 & 0.221 \\
\hline Prior recurrence status (no vs. yes) & 3.997 & 1.175 & 13.602 & 0.027 \\
\hline $\operatorname{NLR}(\leqq 2.29$ vs. $>2.29)$ & 5.911 & 1.579 & 22.126 & 0.008 \\
\hline
\end{tabular}

Kaplan-Meier curve analyses with a log-rank test are shown in Figs. 2, 3, and 4. A high NLR (>2.29) were associated with significantly low RFS and PFS $(P<0.001, P=0.002)$ (Fig. 2). A high CUETO was associated with a significantly low RFS $(P<0.001)$, but there was no significant association between CUETO scores and PFS ( $P=0.423$ ) (Fig. 3 ). After combining NLRs (cutoff value $=2.29$ ) and CUETO scoring (cut-off value $=7$ ), the modified risk model showed that high NLR and CUETO scores were significantly associated with low RFS and PFS $(P<0.001, P=0.002)$ (Fig. 4).

\section{Discussion}

This study identified NLR as a significant factor for predicting tumor recurrence and progression, and inclusion of preoperative NLR enhanced the accuracy of the CUETO model to predict progression in patients with NMIBC.

In the early stages, NMIBC is not life-threatening, but it will recur in more than half of patients and progress from $10-20 \%$ to MIBC [13]. Although numerous efforts have been made to predict and prevent tumor recurrence and progression, the exact characteristics of NMIBC are unknown due to its heterogeneity [14].

The European Organization for Research and Treatment of Cancer (EORTC) has developed a simple scoring system that uses information such as tumor size and number, prior recurrence rate, stage, and concomitant CIS and WHO grade based on data of 2596 patients with NMIBC, to predict the risk of relapse and progression[15]. The CUETO scoring model was created to compensate for the EORTC with low rates of BCG treatment, using information from 1062 patients who received BCG treatment [7]. 
Compared to EORTC, where most of the $78 \%$ patients received intravesical chemotherapy, all patients in the CUETO study received BCG instillation, and $15 \%$ of them received mitomycin C.

Intravesical instillation of BCG is a standard treatment for CIS and an adjuvant option for T1 and highergrade Ta bladder tumors after TUR [16]. The CUETO model is thought to be more suitable for patients treated with BCG. In this study, as with the CUETO study, we included patients who completed 6 BCG instillations. However, compared with the CUETO scoring model, only female gender, concomitant CIS, multiplicity $(>3)$, and prior recurrence status were significant factors for predicting tumor recurrence (all $\mathrm{P}$ $<0.05)$. About tumor progression, only concomitant $\mathrm{CIS}$ and prior recurrence status were significant factors (all $P<0.05$ ). A Kaplan-Meier curve analysis demonstrated that the CUETO score was associated with RFS $(P<0.05)$, but not with PFS $(P=0.423)$. We therefore decided to add the NLR ratio to the CUETO scoring model if inclusion of an NLR would enhance the predictability of CUETO scoring.

Preoperative NLR has proven to be a useful marker and a high NLR has been linked to higher tumor stages and adverse oncologic outcomes in numerous cancers, including not only the gastrointestinal cancer but genitourinary tract cancer, such as urothelial carcinoma of the bladder [17-20]. Although the pathophysiology is not understood clearly, relative neutrophilia may increase inflammatory markers that include proangiogenic factors, growth factors, proteases, and antiapoptotic markers, which facilitate tumor growth and progression [21]. In addition, lymphocytopenia may destroy cell-mediated immune responses and therefore worsen prognoses [22]. In bladder tumors, several previous studies have evaluated the predictive value of NLRs; most focused on MIBC and were conducted mainly on patients who underwent radical cystectomy [23-26]. In 2014, Viers et al. evaluated 899 patients who underwent radical cystectomy without neo-adjuvant chemotherapy and who had a preoperative NLR. An elevated preoperative NLR ( $>2.7$ ) was associated with a significantly higher risk of a locally advanced disease as well as subsequent disease recurrence and cancer-specific and all-cause mortality. In 2012, Can et al. demonstrated that among 80 NMIBC patients and 102 patients with MIBC, an NLR > 2.57 was a predictor of invasive urothelial carcinoma. According to a 2014 study by Potretzke et al., among 102 consecutive patients undergoing radical cystectomy, NLR was significantly related to pathologic tumor staging and to upstaging of non-organ confined disease ( $\geqq$ pT3). Similarly, Krane et al. reported that, among 68 consecutive cases of radical cystectomy for MIBC, an NLR > 2.5 was associated with poor overall and cancer-specific survival, suggesting that such patients may benefit from neo-adjuvant chemotherapy.

When focusing on NMIBC, several trials $[8,17,27,28]$ evaluated the predictive value of the NLR. In 2015, Mano et al. revealed that an NLR $>2.41$ was an independent predictor of disease progression and recurrence in 107 patients with NMIBC treated with TUR. According to Favilla et al.'s study in 2016, an NLR $\geq 3$ was associated with worse disease recurrence $(H R, 2.84 ; P<0.01)$ in 178 patients with Ta or T1 bladder tumor who underwent TUR. The 5 -year RFS was $49 \%$ and $62 \%$ in patients with an NLR $\geq 3$ and < $3(P<0.01)$. A prospective study of Albayrak et al. in 2016 found that a higher NLR was associated with recurrence and progression of Ta or Ta bladder tumors, although, and in contrast with the finding of previous studies, a significant relationship with NLR was lost after correcting for age. Another prospective trial by Getzler et al. in 2018 demonstrated that an NLR $>2.5$ was a significant predictor of disease 
recurrence and a worse RFS in 113 patients with NMIBC, particularly those treated with BCG. As with the studies described above, we found that an NLR > 2.29 was associated with higher tumor recurrence (HR, $2.451 ; 95 \% \mathrm{Cl}, 1.567-3.834 ; \mathrm{P}<0.001)$ and tumor progression $(\mathrm{HR}, 5.911 ; 95 \% \mathrm{Cl}, 1.579-22.126 ; \mathrm{P}=$ 0.008 ) according to a multivariate Cox proportional hazards regression model. Kaplan-Meier curve analysis showed that an NLR $>2.29$ showed significantly low RFS and PFS $(P<0.001$ and $<0.002$, respectively). However, the four studies mentioned above were not restricted to patients treated with BCG.

When narrowing the scope of predictive values of NLRs in all BCG treated patients, Racioppi et al. (2019) [29] evaluated whether an NLR ratio can predict the response to BCG in high-risk NMIBC patients. One hundred consecutive patients with newly diagnosed high-risk NMIBC were analyzed retrospectively. All received an induction course of intravesical immunotherapy with BCG followed by a maintenance course for at least a year. Forty-eight patients underwent radical cystectomy for high-grade recurrence or progression to muscle invasive disease (BCG non-responder group). The mean NLR was $2.61 \pm 0.77$ in the $B C G$ responder group and $3.65 \pm 1.16$ in the $B C G$ non-responder group $(P=0.01)$. The NLR was associated with both recurrence $(P=0.01)$ and progression $(P=0.01)$. A Kaplan-Meier analysis with a log-rank test showed statistically significant differences between the curves for an NLR $<3$ and an NLR $\geq$ $3(P<0.05)$.

Based on the ability of the NLR to predict tumor recurrence and progression, we added the NLR to the CUETO scoring model. Using the CUETO scoring model alone, a significant association was observed with low RFS ( $P<0.001)$, but not with PFS $(P=0.423)$ (Fig. 2). However, after combining the NLR (cut-off value 2.29) and CUETO scoring model (cut-off value 7), the resulting modified risk model showed that a high NLR and high CUETO score were significantly associated with both low RFS and PFS $(P<0.001$ and $P=0.002$, respectively) (Fig. 3). Other combined risk models have been shown to enhance the predictability of each risk model $[8,22]$. Getzler et al. provided statistical evidence that an NLR $>2.5$ may improve the predictive power of an EORTC score when the two are calculated together. In 2019, Aydin et al. evaluated the correlation between NLR and EORTC recurrence and progression scores. They reported that as the NLR increased, recurrence $(P<0.001)$ and progression $(P=0.034)$ scores increased significantly. Nevertheless, this study is the first to analyze the prognostic significance of the NLR and its synergic relation with the CUETO scoring model in patients with NMIBC after intravesical BCG instillation.

There were several limitations to be considered in this study. First, it was based on a retrospective analysis of the records of patients treated at a single institution with unavoidable selection biases. Small numbers and heterogeneous patients are also weak points. It should also be noted that in many previous studies, various NLR cut-off values were evaluated and utilized [30]. Each study's results should be interpreted carefully. Because the idealized and generalized NLR have not yet been established, each study selected cut-off values with different sensitivities and specificities. Furthermore, the main limitation concerning NLRs is the volatile counts of neutrophils and lymphocytes. Although we excluded patients with hematologic malignances and acute or chronic infections, it is possible that individual chronic medications, herbs, or antibiotics affected the NLR value. A prospective study with a larger cohort is 
required to solidify the place of NLR in predicting disease recurrence and progression in patients with NMIBC.

\section{Conclusion}

Our study showed that in patients with NMIBC, the NLR was identified as a significant factor for predicting tumor recurrence and progression. Furthermore, inclusion of a preoperative NLR enhanced the accuracy of the CUETO model to predict disease progression. We therefore recommend that patients with a high NLR receive more aggressive management.

\section{Abbreviations}

BMl: body mass index

CIS: carcinoma in situ

CT: computed tomography

CUETO: Club Urológico Español de Tratamiento Oncológico

EORTC: European Organization for Research and Treatment of Cancer

MIBC: muscle invasive bladder cancer

NLR: neutrophil-to-lymphocyte ratio

NMIBC: nNon-muscle invasive bladder cancer

PFS: progression-free survival

RFS: recurrence-free survival

WHO: World Health Organization

\section{Declarations}

\section{Ethics approval and consent to participate}

This study was approved by the Institutional Review Board of Kyungpook National University Chilgok Hospital (approval number: KNUH 2020-03-042).

\section{Consent for Publication}

Not applicable 


\section{Informed consent}

The institutional review board of Kyungpook National University Chilgok Hospital waived because of the retrospective nature of the study.

\section{Availability of data and material}

The datasets used and/or analyzed during the current study are available from the corresponding author on reasonable request.

All data generated or analyzed during this study are included in this published article.

\section{Competing interests}

The authors declare that there are no conflicts of interest.

\section{Funding}

This research was supported by the Basic Science Research Program through the National Research Foundation of Korea (NRF), and was funded by the Korean Government (MSIT) (2016R1C1B1011180), (2019R1H1A1079839), (2019R1F1A1044473), (2019R1A2C1004046), (2018R1C1B5040264), (2020R1A2B5B03002344) and (2020R1/1A3071568).

\section{Authors' contributions}

JWK: Draft or revision article

JWC: Draft or revision article

EHL: Acquisition of data

SYC: Acquisition of data

DJP: Acquisition of data

KHB: Acquisition of data

SHC: Data analysis

JNL: Data analysis

BSK: Data analysis

HTK: Interpretation of data

ESY: Interpretation of data 
TGK: Interpretation of data

YSH: Conception and design

THK: Conception and design

All authors have read and approved the manuscript.

Acknowledgements

Not Applicable

\section{References}

1. Ferlay J, Soerjomataram I, Dikshit R, Eser S, Mathers C, Rebelo M, Parkin DM, Forman D, Bray F: Cancer incidence and mortality worldwide: sources, methods and major patterns in GLOBOCAN 2012. Int J Cancer 2015, 136(5):E359-386.

2. Babjuk M, Burger M, Zigeuner R, Shariat SF, van Rhijn BW, Comperat E, Sylvester RJ, Kaasinen E, Bohle A, Palou Redorta $\mathrm{J}$ et al: EAU guidelines on non-muscle-invasive urothelial carcinoma of the bladder: update 2013. Eur Urol 2013, 64(4):639-653.

3. Babjuk M, Bohle A, Burger M, Capoun O, Cohen D, Comperat EM, Hernandez V, Kaasinen E, Palou J, Roupret $\mathrm{M}$ et al: EAU Guidelines on Non-Muscle-invasive Urothelial Carcinoma of the Bladder: Update 2016. Eur Urol 2017, 71(3):447-461.

4. Malmstrom PU, Sylvester RJ, Crawford DE, Friedrich M, Krege S, Rintala E, Solsona E, Di Stasi SM, Witjes JA: An individual patient data meta-analysis of the long-term outcome of randomised studies comparing intravesical mitomycin $\mathrm{C}$ versus bacillus Calmette-Guerin for non-muscle-invasive bladder cancer. Eur Urol 2009, 56(2):247-256.

5. Cambier S, Sylvester RJ, Collette L, Gontero P, Brausi MA, van Andel G, Kirkels WJ, Silva FC, Oosterlinck W, Prescott S et al: EORTC Nomograms and Risk Groups for Predicting Recurrence, Progression, and Disease-specific and Overall Survival in Non-Muscle-invasive Stage Ta-T1 Urothelial Bladder Cancer Patients Treated with 1-3 Years of Maintenance Bacillus Calmette-Guerin. Eur Urol 2016, 69(1):60-69.

6. Hidas G, Pode D, Shapiro A, Katz R, Appelbaum L, Pizov G, Zorn KC, Landau EH, Duvdevani M, Gofrit ON: The natural history of secondary muscle-invasive bladder cancer. BMC Urol 2013, 13:23.

7. Fernandez-Gomez J, Madero R, Solsona E, Unda M, Martinez-Pineiro L, Gonzalez M, Portillo J, Ojea A, Pertusa $\mathrm{C}$, Rodriguez-Molina $\mathrm{J}$ et al: Predicting nonmuscle invasive bladder cancer recurrence and progression in patients treated with bacillus Calmette-Guerin: the CUETO scoring model. $J$ Uro/2009, 182(5):2195-2203.

8. Getzler I, Bahouth Z, Nativ O, Rubinstein J, Halachmi S: Preoperative neutrophil to lymphocyte ratio improves recurrence prediction of non-muscle invasive bladder cancer. $B M C$ Urol 2018, 18(1):90. 
9. Lee BS, Lee SH, Son JH, Jang DK, Chung KH, Lee YS, Paik WH, Ryu JK, Kim YT: Neutrophillymphocyte ratio predicts survival in patients with advanced cholangiocarcinoma on chemotherapy. Cancer Immunol Immunother 2016, 65(2):141-150.

10. Viers BR, Thompson RH, Boorjian SA, Lohse CM, Leibovich BC, Tollefson MK: Preoperative neutrophil-lymphocyte ratio predicts death among patients with localized clear cell renal carcinoma undergoing nephrectomy. Urol Oncol-Semin Ori 2014, 32(8):1277-1284.

11. Ceylan C, Doluoglu OG, Keles I, Gazel E, Temucin T, Odabas O, Karalar M: Importance of the neutrophil-to-lymphocyte ratio in muscle-invasive and non-muscle invasive bladder tumors. Urologia 2014, 81(2):120-124.

12. Montironi R, Lopez-Beltran A: The 2004 WHO classification of bladder tumors: a summary and commentary. Int J Surg Pathol 2005, 13(2):143-153.

13. Donat SM: Evaluation and follow-up strategies for superficial bladder cancer. Urol Clin North Am 2003, 30(4):765-776.

14. Choi SY, Ryu JH, Chang IH, Kim TH, Myung SC, Moon YT, Kim KD, Kim JW: Predicting recurrence and progression of non-muscle-invasive bladder cancer in Korean patients: a comparison of the EORTC and CUETO models. Korean J Urol 2014, 55(10):643-649.

15. Sylvester RJ, van der Meijden APM, Oosterlinck W, Witjes JA, Bouffioux C, Denis L, Newling DWW, Kurth K: Predicting recurrence and progression in individual patients with stage Ta T1 bladder cancer using EORTC risk tables: A combined analysis of 2596 patients from seven EORTC trials. European Urology 2006, 49(3):466-477.

16. Lamm DL, Blumenstein BA, Crawford ED, Montie JE, Scardino P, Grossman HB, Stanisic TH, Smith JA, Jr., Sullivan J, Sarosdy MF et al: A randomized trial of intravesical doxorubicin and immunotherapy with bacille Calmette-Guerin for transitional-cell carcinoma of the bladder. N Engl J Med 1991, 325(17):1205-1209.

17. Mano R, Baniel J, Shoshany O, Marge D, Bar-On T, Nativ O, Rubinstein J, Halachmi S: Neutrophil-tolymphocyte ratio predicts progression and recurrence of non-muscle-invasive bladder cancer. Urol Oncol-Semin Ori 2015, 33(2).

18. Chua W, Charles KA, Baracos VE, Clarke SJ: Neutrophil/lymphocyte ratio predicts chemotherapy outcomes in patients with advanced colorectal cancer. Br J Cancer 2011, 104(8):1288-1295.

19. Ku JH, Kang M, Kim HS, Jeong CW, Kwak C, Kim HH: The prognostic value of pretreatment of systemic inflammatory responses in patients with urothelial carcinoma undergoing radical cystectomy. Br J Cancer 2015, 112(3):461-467.

20. Paramanathan A, Saxena A, Morris DL: A systematic review and meta-analysis on the impact of preoperative neutrophil lymphocyte ratio on long term outcomes after curative intent resection of solid tumours. Surg Oncol 2014, 23(1):31-39.

21. Mantovani A, Allavena P, Sica A, Balkwill F: Cancer-related inflammation. Nature 2008, 454(7203):436-444. 
22. Aydin M, Bitkin A, Kadihasanoglu M, Irkilata L, Akgunes E, Keles M, Atilla MK: Correlation of neutrophil-lymphocyte ratio and risk scores in non-muscle invasive bladder cancer. Actas Urol Esp 2019, 43(9):503-508.

23. Viers BR, Boorjian SA, Frank I, Tarrell RF, Thapa P, Karnes RJ, Thompson RH, Tollefson MK: Pretreatment Neutrophil-to-Lymphocyte Ratio Is Associated with Advanced Pathologic Tumor Stage and Increased Cancer-specific Mortality Among Patients with Urothelial Carcinoma of the Bladder Undergoing Radical Cystectomy. European Urology 2014, 66(6):1157-1164.

24. Can C, Baseskioglu B, Yilmaz M, Colak E, Ozen A, Yenilmez A: Pretreatment Parameters Obtained from Peripheral Blood Sample Predicts Invasiveness of Bladder Carcinoma. Urologia Internationalis 2012, 89(4):468-472.

25. Potretzke A, Hillman L, Wong K, Shi FF, Brower R, Mai S, Cetnar JP, Abel EJ, Downs TM: NLR is predictive of upstaging at the time of radical cystectomy for patients with urothelial carcinoma of the bladder. Urol Oncol-Semin Ori 2014, 32(5):631-636.

26. Krane LS, Richards KA, Kader AK, Davis R, Balaji KC, Hemal AK: Preoperative Neutrophil/Lymphocyte Ratio Predicts Overall Survival and Extravesical Disease in Patients Undergoing Radical Cystectomy. $J$ Endouro/ 2013, 27(8):1046-1050.

27. Favilla V, Castelli T, Urzi D, Reale G, Privitera S, Salici A, Russo GI, Cimino S, Morgia G: Neutrophil to lymphocyte ratio, a biomarker in non-muscle invasive bladder cancer: a single-institutional longitudinal study. Int Braz J Urol 2016, 42(4):685-693.

28. Albayrak S, Zengin K, Tanik S, Atar M, Unal SH, Imamoglu MA, Gurdal M: Can the neutrophil-tolymphocyte ratio be used to predict recurrence and progression of non-muscle-invasive bladder cancer? Kaohsiung J Med Sci 2016, 32(6):327-333.

29. Racioppi M, Di Gianfrancesco L, Ragonese M, Palermo G, Sacco E, Bassi PF: Can Neutrophil-toLymphocyte ratio predict the response to BCG in high-risk non muscle invasive bladder cancer? International Braz J Urol 2019, 45(2):315-324.

30. Marchioni M, Primiceri G, Ingrosso M, Filograna R, Castellan P, De Francesco P, Schips L: The Clinical Use of the Neutrophil to Lymphocyte Ratio (NLR) in Urothelial Cancer. A Systematic Review. Clin Genitourin Canc 2016, 14(6):473-484.

\section{Figures}




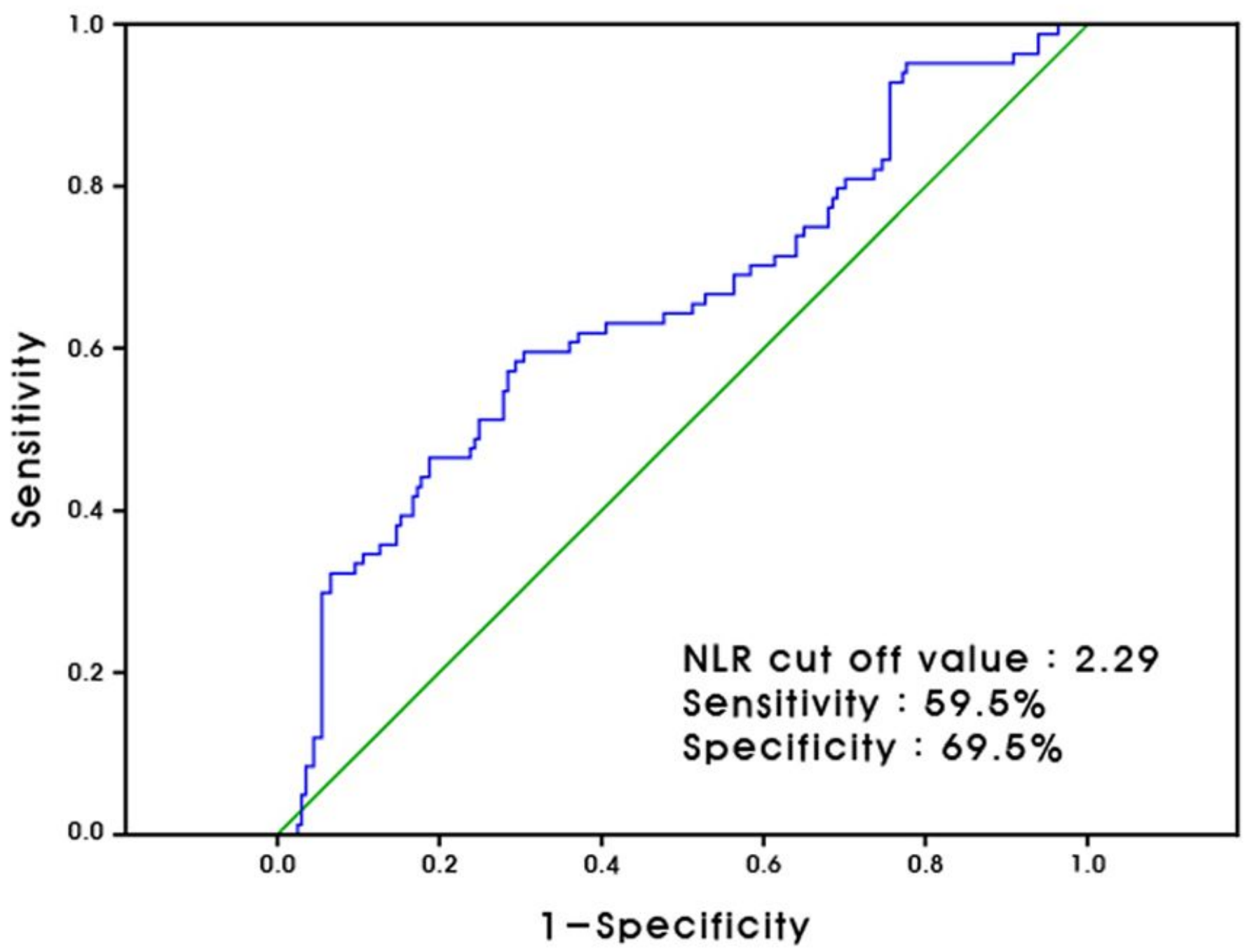

Figure 1

The best cut-off NLR value according to the ROC curve 

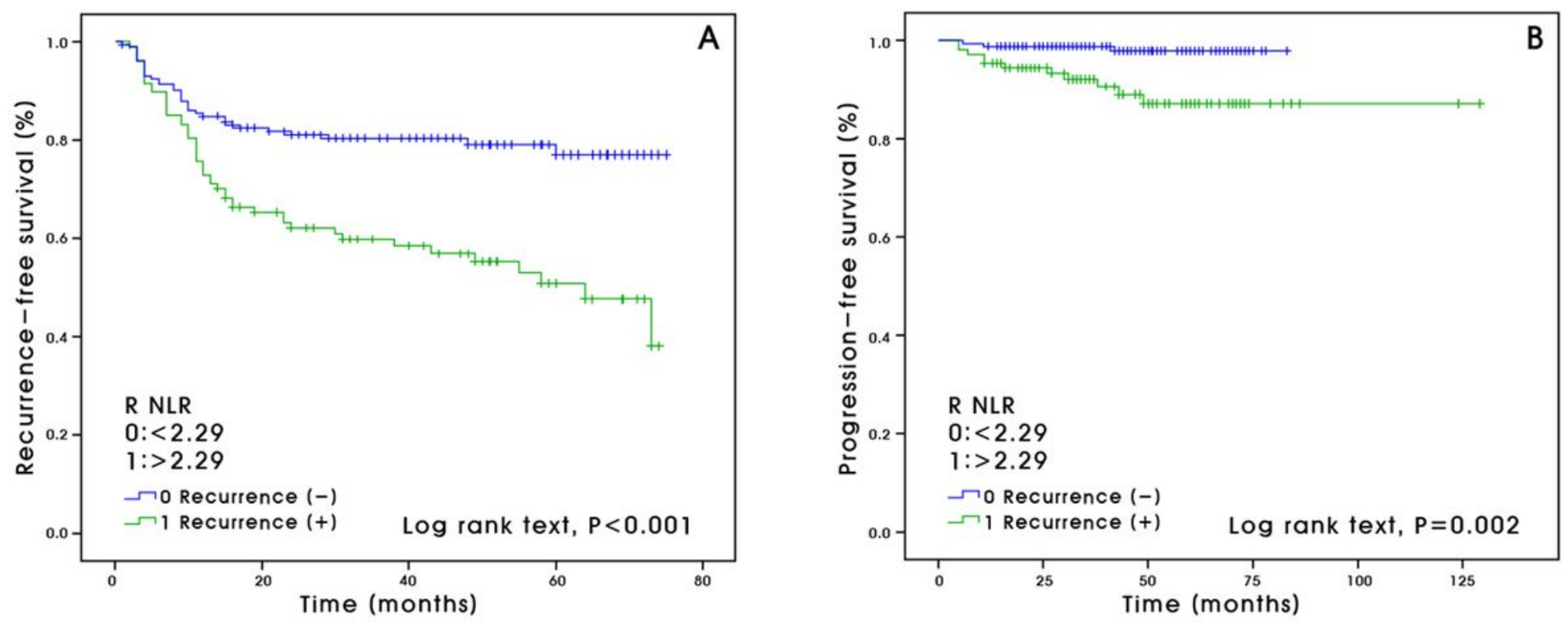

Figure 2

Kaplan-Meier curve analysis for recurrence-free survival (A) and progression-free survival (B), according to NLRs
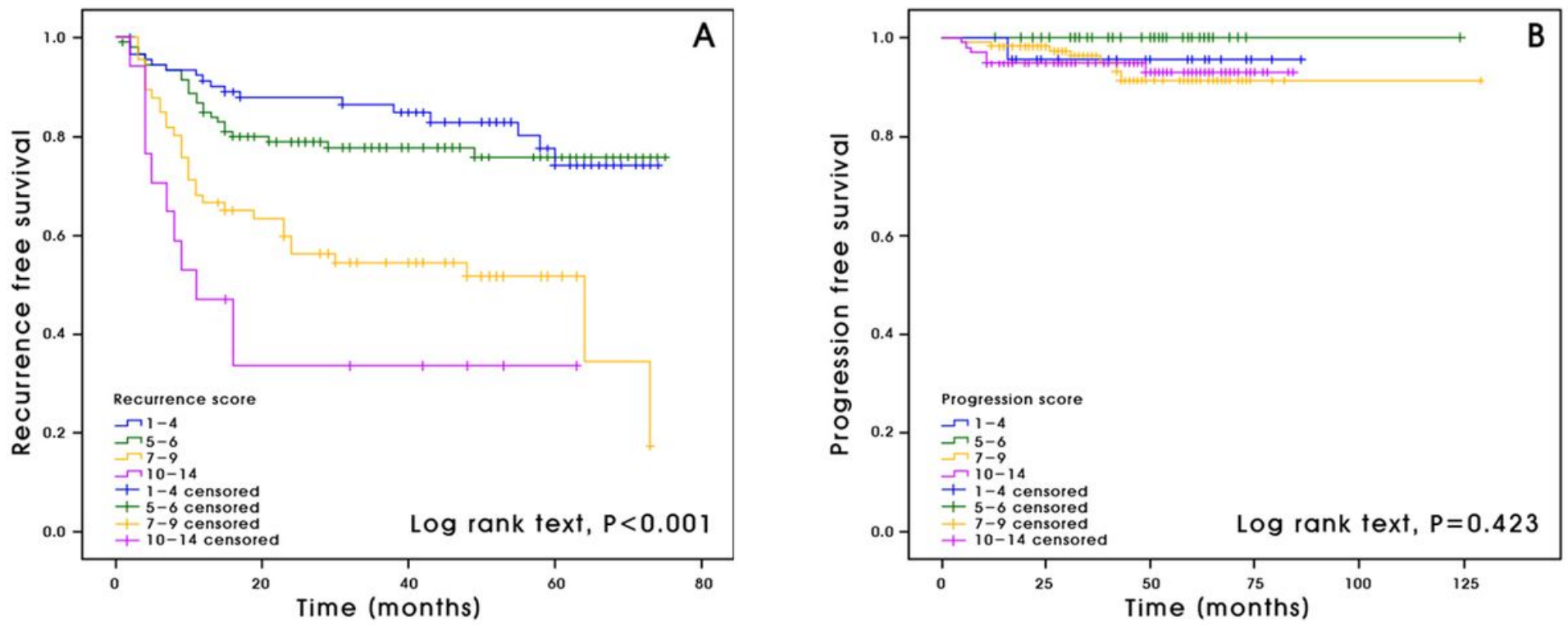

Figure 3

Kaplan-Meier curve analysis for recurrence-free survival (A) and progression-free survival (B), according to CUETO scores 

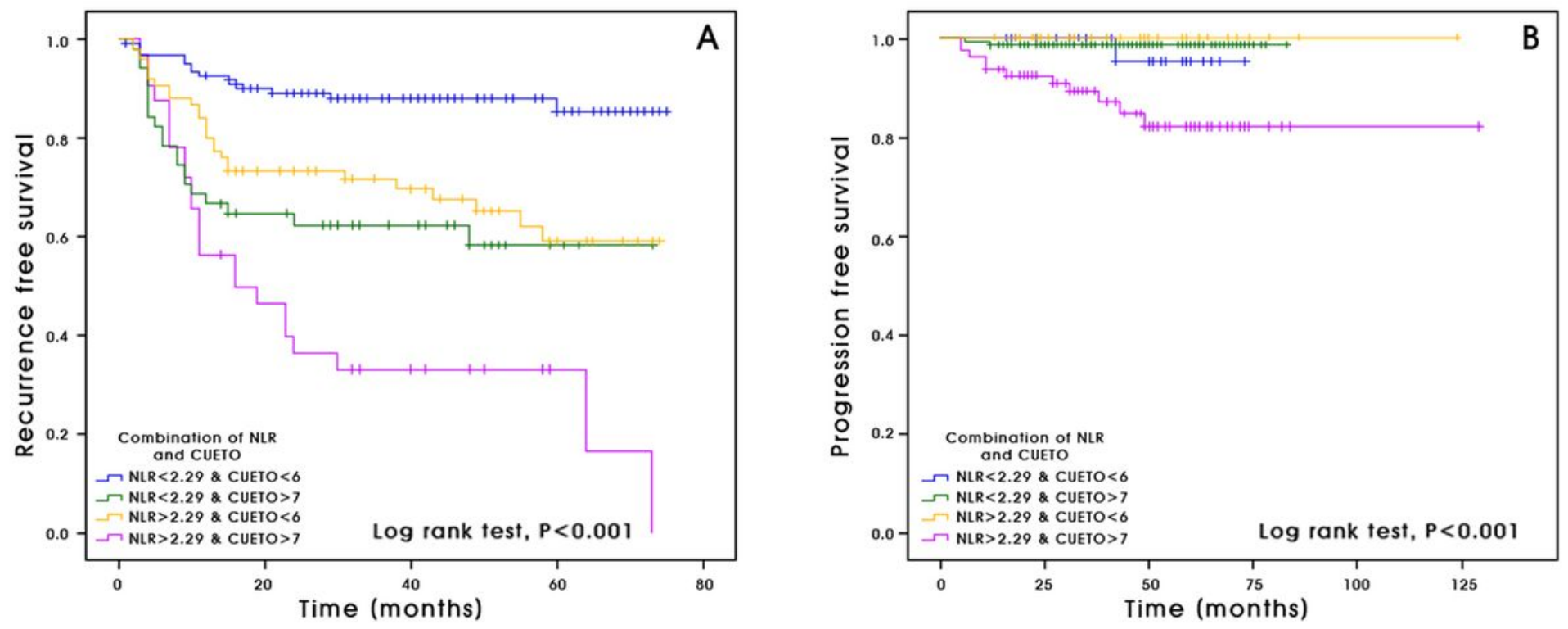

Figure 4

Kaplan-Meier curve analysis for recurrence-free survival (A) and progression-free survival (B), according to a combination of NLRs and CUETO scores 